\title{
La profondità ipocentrale con metodi macrosismici
}

Il valore di $\gamma$ in Italia nella formula $\left(I_{\mathrm{o}}-I_{n}\right)=\gamma \log \left(L_{n} / n\right)$ e limiti di applicabilità del metodo.

(The hypocentral depth by macroseismic methods. The $\gamma$ value in Italy in the formula $\left(I_{\mathrm{o}}-I_{n}\right)=\gamma \log \left(D_{n} / h\right)$ and application limits for the method).

\section{MARCELli * - M.C. SPAdEA *}

Ricevuto il 6 Gennaio 1981

\section{RIASSUNTO}

Scopo del presente lavoro è la determinazione della profondità ipocentrale di un terremoto con metodi macrosismici.

Viene fatta una rapida rassegna storica delle principali relazioni esistenti tra intensità macrosismica, accelerazione, e profondità ipocentrale a partire da Cancani fino all'epoca attuale.

Particolare attenzione è stata posta alle opere di Kovesligethy e di Blake. Essendo la relazione di quest'ultimo alla base di tutti i metodi moderni, si è passati alla ricerca del valore sperimentale di $\gamma$ da applicare in Italia nella formula

$$
\left(I_{\mathrm{o}}-I_{n}\right)=\gamma \log \left(D_{n} / h\right)
$$

facendo uso di un certo numero di terremoti di nota profondità analitica.

$\left.{ }^{\star}\right)$ Istituto Nazionale di Geofisica, Roma. 
Sono stati individuati due valori medi di $\gamma$. Uno $\left(\bar{\gamma}_{g}\right)$ valido per L'Italia in generale

$$
\bar{\gamma}_{s}=4.382 \pm 2.282
$$

e uno $\left(\gamma_{c}\right)$ più specifico per l'Italia centrale

$$
\bar{\gamma}_{c}=3.989+1.344
$$

Dall'esame condotto si ha motivo di ritenere che il metodo macrosismico non sia applicabile indiscriminatamente e si propongono i seguenti limiti di applicabilità:

1) quanto alle isosiste da usare . . (non più di tre intorno all'epicen.)

2) quanto alle profondità ipocentrali (solo entro la crosta: $h<33 \mathrm{~km}$ )

3) quanto al valore di $\gamma . . . .(\gamma<7.0)$

E' stata calcolata una tabella (la 3 ) per una rapida determinazione della profondità $(h+\delta h)$ quando siano stati calcolati (o misurati) i raggi medi $\bar{r}_{c}$ dei cerchi equivalenti alle aree della prima, della seconda e della terza isosista di un terremoto.

Nella tabella 4 sono state poste a confronto le profondità macrosismiche con quelle analitiche per i terremoti utilizzati.

I risultati conseguiti nella presente ricerca sono da ritenersi migliorabili nel momento in cui si potrà contare su un maggior numero di terremoti di nota profondità analitica (associata alla conoscenza del panorama macrosismico) e sulla possibilità di associare ad un valore medio generale $\bar{\gamma}$, valori $\pm \delta \bar{\gamma}_{i}$ specifici per ogni unità strutturale omogenea.

Comunque sia, i risultati che si ottengono con questo, come con altri metodi macrosismici, sono sempre da ritenersi approssimativi, anche se qui si è tentato di dare persino un "range" di indeterminazione ai valori di $h$.

\section{ABSTRACT}

Aim of the present work is to determine the hypocentral depth of an earthquake by macroseismic methods.

A short hystorical review of the main relations between macroseismic intensity, acceleration and hypocentral depth from Cancani to the present 
time has been carried out. Particular care has been pointed out to Kovesligethy and Blake works. Being the Blake's relation the basis of all modern methods the $\gamma$ experimental value has been investigated to apply in Italy in the formula

$$
\left(I_{\mathrm{o}}-I_{n}\right)=\gamma \log \left(D_{n} / h\right)
$$

using some earthquakes with analitical depth known. There are singled of two intermediate values of $\gamma_{i}$ one $\left(\bar{\gamma}_{\alpha}\right)$ valid for Italy in general

$$
\bar{\gamma}_{s}=4.382 \pm 2.282
$$

another $\left(\bar{\gamma}_{c}\right)$ more specific for central Italy

$$
\bar{\gamma}_{c}=3.989 \pm 1.344
$$

From the investigation the AA. deem that the macroseismic method cannot anyhow be applied, hence the AA. suggest the following application limits:

1) for the isoseismals to be used (not more than three around the epic.)

2) for the hypocentral depths (only within the crust: $h<33 \mathrm{~km}$ )

3) for the $\gamma$ value $(\gamma<7.0)$

To determine quickly the depth $(h+\delta h)$, when the intermediate radii $\bar{r}_{c}$ of the circles equivalent to the I, II, III isoseismal areas of an earthquake are been calculated (or measured), a table 3 has been calculated.

In table 4 both the macroseismic and the analitical depths relating to untilized earthquakes, are compared.

The results obtained in the present work can be improved when the researchers will have access to a greater lot of earthquakes with known analitical depth and will be able to associate to a general intermediate value $\bar{\gamma}$, specific values of $\pm \delta \gamma_{i}$ for each homogeneous structural unit.

In any case, the results obtained by that, as well by different macroseismic methods, have always to retain approximate, even if the AA. tried to furnish a range of indetermination to $h$ values. 


\section{INTRODUZIONE}

E' noto che tra i parametri ipocentrali di un terremoto, quello di più difficile determinazione è la profondità ipocentrale $h$. Valori relativamente attendibili si possono ottenere quando se ne determina il valore per via analitica, ed in particolare quando l'analisi dei sismogrammi consente l'individuazione delle onde $p P$ caratteristiche delle profondità ipocentrali. Ma questo avviene molto raramente, anche con le attrezzature strumentali moderne più avanzate, per cui il calcolo delle profondità si fa in genere utilizzando i tempi d'arrivo delle prime onde nei vari Osservatori e procedendo ai calcoli analitici sulla base di alcuni modelli di dromocrone ritenuti più validi.

Sui risultati che si ottengono con procedimento analitico è stato discusso in altra nota (Marcelli, Peronaci, 1979). Qui ci proponiamo di discutere sulla determinazione delle $h$ con metodo macrosismico, metodo dal quale non si può prescindere quando si voglia attribuire un valore della profondità a terremoti privi di qualsiasi documentazione strumentale.

Va subito premesso che qualunque tentativo di calcolo che voglia utilizzare dati macrosismici sarà sempre da ritenersi valido solo in via approssimativa, giacché i dati che vengono utilizzati a tal fine non potranno mai costituire un modello di precisione matematica.

L'impostazione teorica che se ne vuol dare rappresenta solo una guida iniziale per introdurre alcuni concetti fondamentali; il problema però va poi risolto in maniera del tutto sperimentale, e pertanto con un debole supporto teorico.

E' per questo che il metodo macrosismico va usato con le debite riserve: esso ha avuto ed ha tuttora, tra i cultori della sismologia, i suoi sostenitori e i suoi detrattori.

Tuttavia il tentativo di trarre la conoscenza sia pure approssimativa di un parametro tanto importante come è la profondità ipocentrale, può servire ad arricchire i dati di informazione di un catalogo sismico storico e può condurre ad acquisire ulteriori elementi conoscitivi sull'aspetto macrosismico di un terremoto e le sue probabili implicazioni geofisiche. 
1. UNA RASSEGNA STORICA DELLE PRINCIPALI RELAZIONI TRA ALCUNI PARAMETRI SISMICI

Nonostante tante ragionevoli critiche e tante incertezze sulla attendibilità dei metodi macrosismici, il problema, sollevato ai primi del secolo, continua a tener viva l'attenzione dei sismologi di tutto il mondo.

Esponiamo una rapida rassegna storica delle principali relazioni, soffermandoci solo su quelle che riteniamo basilari per il nostro lavoro (Cancani, Kovesligethy, Gassmann, Blake).

Nel 1904 Adolfo Cancani presentò a Lipsia, dove si teneva la seconda Conferenza Internazionale di Sismologia, una sua relazione dal titolo "Sur l'emploi d'une double échelle sismique des

\section{TABELLA A}

Tabella di ragguaglio fra $\mathbf{i}$ valori dell'accelerazione e $\mathrm{i}$ gradi convenzionali della scala Forel-Mercalli

CANCANI

\begin{tabular}{|c|c|c|c|}
\hline $\begin{array}{c}\text { Gradi } \\
\text { della } \\
\text { scala }\end{array}$ & $\begin{array}{l}\text { Denominazione ordinaria } \\
\text { della scala For-Mercalli }\end{array}$ & $\begin{array}{l}\text { Accelerazioni } \\
\mathrm{mm} / \mathrm{sec}^{2}\end{array}$ & $\begin{array}{l}\text { Grablovitz } \\
\quad(1910)\end{array}$ \\
\hline I & Scossa strumentale. & $<2.5$ & - \\
\hline II & Leggerissima . . . . & $2.5-5.0$ & 3.2 \\
\hline III & Leggera . . . . . . & $5-10$ & 6.8 \\
\hline IV & Sensibile o mediocre. & $10-25$ & 14.6 \\
\hline V & Abbastanza forte. . . & $25-50$ & 31.4 \\
\hline VI & Forte . . . . . . . & $50-100$ & 67.5 \\
\hline VII & Fortissima . . . . . & $100-250$ & 145.2 \\
\hline VIII & Rovinosa . . . . . . & $250-500$ & 312.3 \\
\hline IX & Disastrosa . . & $500-1000$ & 671.6 \\
\hline $\mathrm{X}$ & Disastrosissima . . & $1000-2500$ & 1444.5 \\
\hline XI & Catastrofe . . . . . & $2500-5000$ & 3106.9 \\
\hline XII & Enorme catastrofe . . . & $5000-10000$ & 6682.3 \\
\hline
\end{tabular}

N.B. - I gradi XI e XII furono aggiunti dal Cancani in seguito ai terremoti disastrosi di Mino-Owari (Giappone) del 1891, e dell'India del 12-6-1897. 
intensités, empirique et absolute ". Ivi l'autore stabiliva una tabella di ragguaglio tra i valori di accelerazione del suolo e i vari gradi convenzionali della scala delle intensità Forel-Mercalli allora in uso.

Per ogni grado di intensità l'autore dava due limiti dell'accelerazione con la quale il terreno verrebbe sollecitato. (Nel 1910 Grablovitz proponeva per l'accelerazione i valori medi centrali riportati nell'ultima colonna della tabella A).

\section{$1.11906-R$. von Kovesligethy}

Il Cancani morì nell'anno stesso in cui aveva presentato la sua relazione: questa sollevò però l'attenzione dello scienziato ungherese R. von Kovesligethy il quale, nella sua opera latina "Seismonomia" pubblicata nel 1906, riprende il discorso di Cancani per trarne delle conclusioni di grande importanza. Egli intravede nella tabella presentata da Cancani, la possibilità di tradurre in termini analitici quella che in realtà è la "Legge psicofisica di Fechner " $\left(^{*}\right)$ che l'autore ungherese ritiene applicabile anche alla sismologia. Egli pone infatti la seguente relazione tra l'accelerazione massima $\Gamma$ prodotta da una scossa di intensità $G$ e l'intensità stessa:

$$
G=x+y \log \Gamma\left(\Gamma \text { in } \mathrm{mm} / \mathrm{sec}^{2}\right)
$$

Attraverso i valori limite dati dal Cancani, calcola le costanti $x$ e $y$ della equazione, desumendone la seguente relazione finale:

$$
G=3 \log 4 / 3 \mathrm{r}
$$

che in memoria di Cancani chiamerà equazione cancaniana.

(*) La "Legge psico-fisica di Fechner" (del 1860) dice che:

"L'intensità di una sensazione è proporzionale al logaritmo dello stimolo". 
Per una accelerazione pari all'accelerazione di gravità si avrebbe, secondo la formula [1]

$$
G=12.35
$$

Secondo Omori l'uomo avverte l'accelerazione fino al limite di $17 \mathrm{~mm} / \mathrm{sec}^{2}$ cui compete tramite la [1] un grado di intensità

$$
G=4.066
$$

che rappresenta quindi, sempre secondo Omori, il limite inferiore di percettibilità umana.

Dalla [1] lo stesso von Kovesligethy deduce quella relazione che sarà alla base di tutte le ricerche future della profondità ipocentrale in funzione dell'intensità nella ipotesi "sine magnum errandi periculo licebit supponere " che nell'area macrosismica la propagazione avvenga in modo rettilineo (per " area macrosismica " Kovesligethy intende l'area delimitata dalla isosista di IV Rossi-Forel, al limite della percettibilità umana).

In tale ipotesi, dice l'autore, si può supporre che le accelerazioni $\Gamma_{1}$ e $\Gamma_{2}$ in due punti distanti $\Delta_{1}$ e $\Delta_{2}$ dal centro di scuotimento stiano tra loro in un rapporto inverso alle distanze, per cui, chiamata $h$ la profondità ipocentrale, $G_{0}$ l'intensità all'epicentro, $G$ l'intensità del sisma in un punto $A$ distante $r_{n}$ dall'epicentro, $D_{n}$ la distanza di $A$ dall'ipocentro ed $e$ l'angolo di emergenza del flusso dell'energia sismica in $A$, sarà:

$$
\left(G-G_{\mathrm{o}}\right)=3 \log \sin e=3 \log \left(\frac{h}{D_{n}}\right)
$$

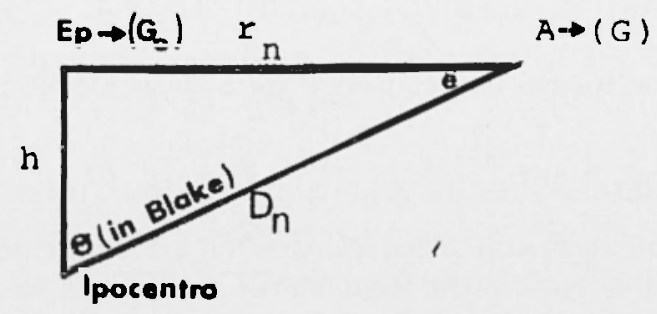


Se poi si tien conto dell'assorbimento del mezzo attraversato dalle onde sismiche, la forma più completa dell'equazione di Kovesligethy sarà:

$$
\left(G-G_{0}\right)=3 \log \sin e-3 \alpha \frac{D_{n}}{R}(1-\sin e) \log \varepsilon
$$

essendo $\alpha$ il coefficiente di assorbimento per unità di tragitto (sperimentale)

$R$ il raggio terrestre

$\varepsilon$ la base dei logaritmi naturali

La [3] ovviamente si può scrivere anche $\left({ }^{*}\right)$

$$
\left(G_{\mathrm{o}}-G\right)=3 \log \left(\frac{D_{n}}{h}\right)+3 \alpha \log \varepsilon \frac{1}{R}\left(D_{n}-h\right)
$$

Lo stesso anno (1906) il sismologo ungherese Jánosy (1906) si servì dell'equazione proposta da Kovesligethy per studiare alcuni terremoti avvenuti a Charleston (profondità ipocentrale e coefficiente di assorbimento), combinando però le varie isosiste fra loro in modo da escludere dai calcoli il valore della intensità massima all'epicentro.

Successivamente molti altri si sono serviti della stessa formula introducendo alcune modifiche inessenziali. Ne citiamo alcuni: Grablovitz (1910) che però polarizza la sua attenzione prevalentemente sulle accelerazioni; Steikoff (1912); Negri (1913); Oddone (1908, 1915, 1930); Inglada (1921, 1950); Martinelli (1923, 1931); Gassmann (1925) (vedi oltre); Neuberger (1938); Caloi (1939); Blake (1941), di cui parleremo più in dettaglio tra breve;

(*) N.B. - La forma più consueta sotto la quale si trova la [3] è:

$$
\left(I_{\mathrm{o}}-I_{\mathrm{n}}\right)=3 \log \left(\frac{D_{n}}{h}\right)+\beta\left(D_{n}-h\right)
$$

dove $\beta=(3 \log \varepsilon) k$, con $k$ (coefficiente di assorbimento che vale $\alpha / R$ della [3']) variabile a seconda degli autori (in genere va da 0.00 a 0.06 ). 
Gutenberg e Richter (1942, 1948, 1956); Galanopoulos (1950); Imbò e Casertano (1952); Peterschmitt (1952, 1963), 1964, 1969); Neumann (1954); Raimondi (1956); Shebalin (1957, 1959, 1961); Sponheuer (1958, 1960, 1971); Medvedev (1959); Bath (1960); Mei-ShiYun, C.Y. Fu, C.I.Liu (1960-61); Karnik (1961, 1963, 1965, 1969); Shenkareva (1971, 1979); Rothé (1972); Carrozzo-De VisintiniGiorgetti (1973); Peronaci; Iaccarino; Bottari-Federico-Lo Giudice (1979), e numerosi altri, italiani e non, che hanno utilizzato ed utilizzano tuttora formule derivanti dalla Cancani-Kovesligethy.

\subsection{5 - La formula di Gassmann}

Nel 1925 Gassmann, indipendentemente da Kovesligethy, aveva proposto la seguente formula:

$$
\frac{J-j}{3}=\log _{10} \sqrt{1+\left(R_{i} / h\right)^{2}}+q h\left(l^{i} 1+\left(R_{i} / h\right)^{2}-1\right)
$$

e se ne era servito per studiare i terremoti del Wallis dell'aprile 1924.

Si noti però che la [4] è sostanzialmente identica alla [3'] di Kovesligethy $\left(J=\right.$ intensità massima; $R_{1}=$ raggio dell'area racchiusa dall'isosista $j_{m a} ; h=$ profondità ipocentrale; e $q$ è un coefficiente d'assorbimento empirico confrontabile con l'a.

\subsection{1 - La formula di Blake}

In un lavoro del 1941 Blake ha tentato di applicare la formula di Kovesligethy-Gassmann ad uno studio sul terremoto del Montana dell'ottobre 1935, usando per il coefficiente di assorbimento $q$ il valore che Gassmann aveva trovato per la zona del Wallis; ma i valori che ne trae non lo soddisfano e quindi ne deduce che $q$ non possa ritenersi costante. Non si può sperare, dice l'autore, di trovare un valore costante dell'assorbimento là 
dove le strutture del terreno attraversato dalle onde sismiche non siano omogenee: il coefficiente di assorbimento dovrebbe quindi essere fornito in base al confronto con terremoti di cui si conoscano le profondità ipocentrali dedotte strumentalmente.

L'autore ritiene che comunque andrebbe cercata un'altra legge di variazione, più idonea di quella di KovesligethyGassmann, per dare una fornula da usare per la stima di $h$ e suggerisce l'uso di un'altra formula empirica (quella che sarà alla base di tutta la nota presente) fondata prevalentemente sui due presupposti fondamentali seguenti:

a) la validità della relazione proposta dal Cancani secondo cui l'accelerazione prodotta da un terremoto in un punto in cui l'intensità valga $j$, è proporzionale a $10^{1 / 3}$;

b) propagazione rettilinea dei raggi sismici. Ammesso poi che la distruzione provocata da un terremoto sia dovuta principalmente alla componente orizzontale del moto e che pertanto le onde $S$ siano le principali responsabili del trasporto dell'energia distruttiva, ad una certa distanza dall'epicentro l'energia associata alle $S$ sarà ovviamente solo una parte di quella all'epicentro; e questa porzione, nell'ipotesi di propagazione rettilinea dei raggi, dipenderà dal coseno dell'angolo $\theta$ con cui l'onda sismica emerge dal fuoco. (Questo coseno rappresenta il rapporto tra il flusso d'energia sulla superficie della terra e il flusso sulla superficie della sfera di raggio $D$ e centro nel fuoco: $\theta=\operatorname{arctg}$ $\left.R_{i} / h\right)$.

Il coseno comparirà come potenza $r_{\text {estma }}$ (con $r$ variabile da 0 ad 1) ossia

$$
10^{i / 3} \propto\left(1 / D \cos ^{\gamma} \theta\right)
$$

In base a questi presupposti Blake propone la formula

$$
J-j=-s \log _{10} \cos \theta
$$

avendo posto $s=3(1+r)$ con $r$ che può assumere valori compresi tra 0 e 1 (pertanto $s$ assumerà valori compresi fra 3 e 6). 
Se adottiamo la simbologia usata per la formula di Kovesligethy, si vede che la [6], pur essendo stata ottenuta partendo da presupposti non identici, assume l'aspetto

$$
I_{0}-I_{n}=s \log _{10}\left(D_{n} / h\right)
$$

la [6'] è formalmente uguale alla [3'] di Kovesligethy quando in questa ultima venga trascurato il secondo termine del secondo membro

$$
3 x \log \varepsilon \frac{1}{R}(D-h)
$$

Nella [6'] però è fondamentale la conoscenza di $s$ che solo in casi particolari puo assumere il valore " 3 " che si ritrova invece come termine fisso nelle formule di Kovesligethy e di Gassmann.

A detta dell'autore (Blake) una trattazione analitica del problema andrebbe affrontata in modo molto più complesso, con l'introduzione di ulteriori parametri (l'assorbimento varia sensibilmente con la profondità; si dovrebbero introdurre le variazioni elastiche subite dalle varie rocce per effetto delle tensioni interne; si dovrebbe tener conto della propagazione discontinua dell'energia sismica, nonché del fatto che la rottura all'ipocentro non è mai puntiforme ecc.) ma la difficoltà di tradurre in termini matematici elementi così aleatori e di difficile precisazione è tale da rendere praticamente giustificata una ricerca di curve sperimentali. D'altronde, conclude l'autore (e noi con lui) il meglio che ci si può aspettare da dati non strumentali è solo una stima approssimata della profondità ipocentrale.

Quasi tutti gli autori che hanno affrontato il problema della determinazione di $h$ con metodi macrosismici si sono serviti, con varianti più formali che sostanziali della relazione di Kovesligethy o di quella di Blake. del tipo

In tempi recenti alcuni di essi hanno anche usato relazioni

$$
\begin{aligned}
M & =\alpha I_{0}+\log h+\beta \quad \text { oppure del tipo } \\
\log E & =a I_{0}+b \log h+c
\end{aligned}
$$


ma l'uso di esse (anche ammettendo noti i valori delle costanti $\alpha, \beta, \circ a, b, c)$ presuppone la conoscenza della magnitudo $M \circ$ dell'energia $E$, elementi entrambi da valutarsi strumentalmente, l'uno funzione dell'altro.

Relazioni di quest'ultimo tipo vanno escluse dalla presente indagine giacché lo scopo precipuo della nostra ricerca è quello di poter valutare la profondità ipocentrale con dati esclusivamente macrosismici.

\section{RicERCA DEL $\gamma$ IN ITALIA}

Il punto principale del nostro problema è pertanto la ricerca sperimentale di un valore del coefficiente $s$ (che compare nella $\left.\left[6^{\prime}\right]\right)$, valido per i terremoti con epicentro sul territorio italiano.

Conformemente alle simbologie correnti, posto $s=\gamma$, la formula di Blake nella forma

$$
\left(I \circ-I_{n}\right)=\gamma \log \left(\frac{D_{n}}{h}\right)
$$

è stata usata da molti autori per la ricerca di valori di $\hat{i}$ in varie parti del mondo.

Schematizziamo nella tabella 1 alcuni valori di $\gamma$.

La tabella mostra quanto sia esteso l'intervallo di variazione del $\gamma$. Pertanto la scelta di un determinato valore di esso influisce notevolmente sul valore della profondità ipocentrale desunta con metodo macrosismico.

L'ipotesi della propagazione rettilinea dei raggi sismici assunta da Kovesligethy prima e da Blake poi, riduce il campo di applicabilità della formula. Non si possono superare certe distanze epicentrali senza annullare tale ipotesi, per cui il valore di $\gamma$ risulta fondamentalmente legato alla struttura della crosta in prossimità dell'epicentro, e poiché tale struttura è variamente eterogenea da regione a regione, ne consegue che è da ritenere troppo semplicistico assumere per $\gamma$ un valore unico come invece 


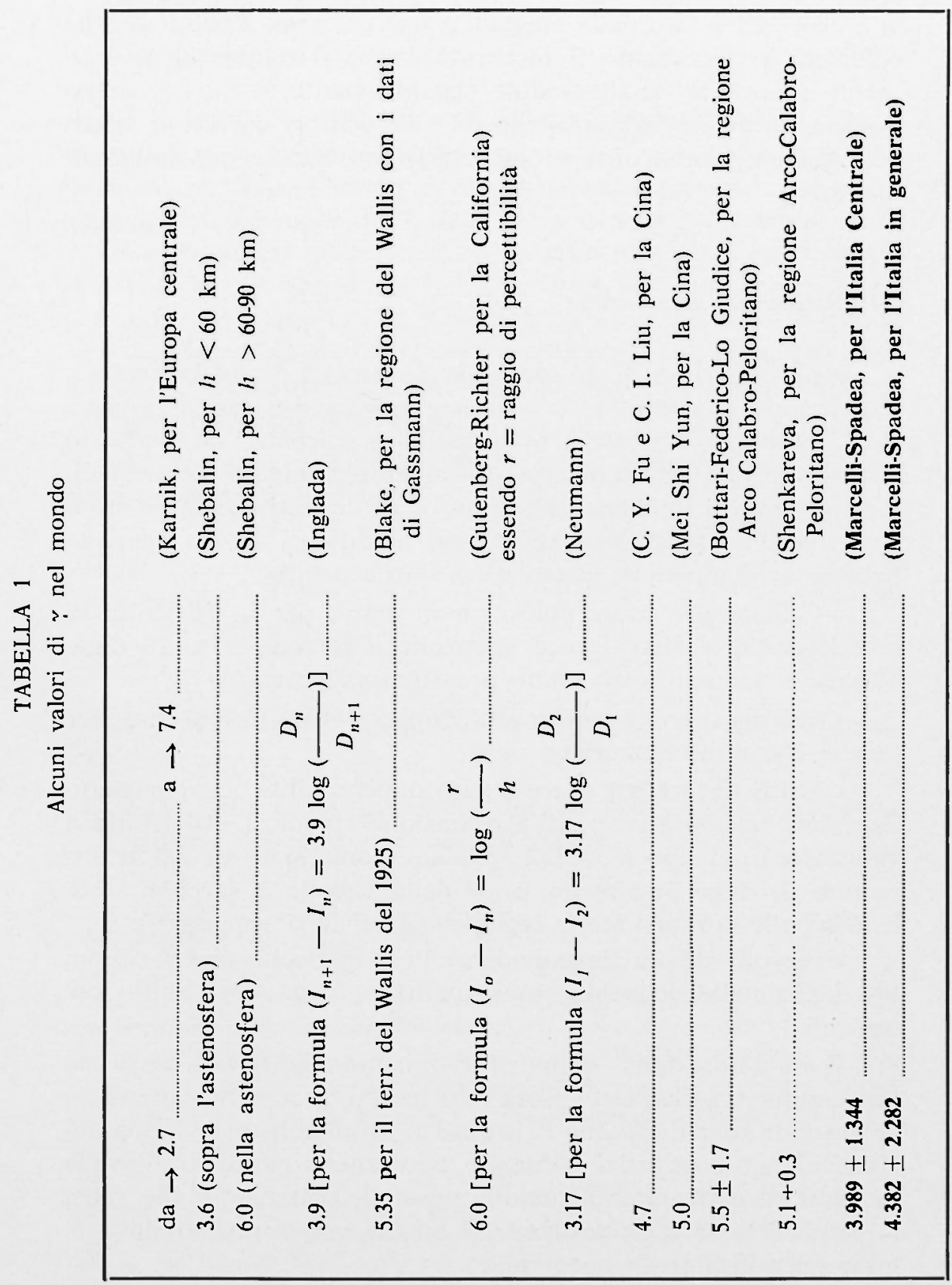


si è costretti a fare nella maggior parte dei casi. Esso andrebbe calcolato suddividendo il territorio in parti minori strutturalmente omogenee, analizzandole separatamente, o quanto meno associando ad un valore medio di $\gamma$ dei fattori correttivi adatti ad ogni singola struttura omogenea; ma torneremo sull'argomento.

\subsection{Scelta dei terremoti}

Nella difficoltà di procedere ad un'analisi dettagliata abbiamo ritenuto più opportuno condurre la ricerca a carattere generale. A tal fine, fra tutti i terremoti con epicentro su territorio italiano documentabili macrosismicamente, abbiamo scelto quelli i cui parametri fondamentali erano stati desunti mediante studi particolari o mediante elaborazioni analitiche. In un secondo tempo anche alcuni di questi sono stati scartati:

- alcuni per avere epicentro in mare, per la difficoltà di tracciarne le isosiste (unica eccezione il terremoto n. 18 della Tabella 2 con epicentro molto prossimo alle coste);

- altri per avere profondità troppo elevate $(h>33 \mathrm{~km})$, per motivi che puntualizzeremo oltre;

- altri ancora per avere il dato di profondità accompagnato da valori troppo elevati della deviazione standard. (Di fronte a valutazioni del tipo $h=(22+39) \mathrm{km}$, come si trova per il terremoto del lago Trasimeno, n. 34 della Tabella 2, sarebbe stato ben difficile fare una scelta ragionevole per la ricerca dei $\gamma$ ).

Una volta di più viene evidenziata la difficoltà che si riscontra anche nelle determinazioni analitiche delle profondità ipocentrali.

$E^{\prime}$ da domandarsi: se un procedimento analitico-strumentale può condurre a risultati ancora così incerti, perché non prendere in maggior considerazione $\mathrm{i}$ risultati raggiungibili per via macrosismica? non sostitutivi, s'intende, ma almeno paralleli, in modo da renderli confrontabili quando possibile. Riteniamo che valga la pena di tentare un recupero di fiducia verso un metodo ritenuto scientificamente poco valido. 


\subsection{Metodologia della ricerca}

I terremoti scelti sono stati studiati pertanto anche macrosismicamente: disegnate le isosiste successive alla intensità massima, se ne sono misurate le aree e calcolati i raggi $\bar{r}_{c}$ dei cerchi equivalenti. Nel caso di isosiste aperte (come per esempio sulle coste o ai confini) i raggi medi sono stati « misurati » sul disegno: un tentativo di introdurre nei calcoli anche i raggi di massima e minima propagazione di intensità è stato abbandonato perché si è rivelato superfluo e dispersivo; una sintesi mediata dei risultati riconduce a valori quasi identici a quelli conseguibili con i raggi dei cerchi equivalenti.

La formula di Blake è stata usata esplicitandone la profondità ipocentrale

$$
h=\frac{r_{i}}{\sqrt{10 \frac{2\left(I_{0}-I_{2}\right)}{\gamma}-1}}
$$

la [8] è la forma comunemente usata per una rapida determinazione di $h$.

La Tabella 2 riporta l'elenco dei terremoti, i dati di partenza, e i risultati delle elaborazioni successive.

Alcuni tentativi preliminari per la ricerca del $\gamma$

Accenniamo brevemente ad alcuni sondaggi preliminari intrapresi per un buon uso della relazione di Blake.

- Un primo approccio di carattere estremamente generale è stato fatto con l'uso del calcolatore opportunamente programmato ("). Questo tentativo ci ha condotto a ritenere il metodo applicabile solo entro certi limiti di profondità.

(*) A questo tipo preliminare di programmazione ha collaborato il Dr. B. De Simoni dell'Y.N.G. 
Infatti per $h>30 \div 33 \mathrm{~km}$ il valore di $\gamma$ diventa in generale talmente elevato da non essere più ragionevolmente accettabile se si vuol far uso della formula di Blake nella forma originale:

$$
J-j=-s \log _{\omega} \cos 0
$$

in quanto Blake dice esplicitamente che vale la posizione seguente per $s$ ( $\gamma$ per noi)

$$
s=3(1+r) \quad \text { con } 0<r<1
$$

per cui $s$ assumerà valori compresi fra 3 e 6 ; quindi valori troppo elevati di $\gamma$ andrebbero esclusi come infatti vedremo oltre.

- La relazione di Blake è stata poi utilizzata per trovare il valore di $\gamma$ in vari modi:

a) utilizzando solo i dati della prima isosista

b) utilizzando solo i dati delle prime due isosiste

c) utilizzando i dati delle prime tre isosiste

d) utilizzando i dati di tutte le isosiste disponibili:

la scelta, come vedremo, è caduta sul tentativo c).

Questi conti effettuati per tutti i terremoti, compresi quelli a grande profondità (ma questi ultimi con le sole prime tre isosiste) danno valori di $\bar{\gamma}$ troppo elevati.

I risultati migliorano considerando solo i terremoti con $h<33 \mathrm{~km}$ per i quali il $\bar{\gamma}$ risulta contenuto entro limiti accettabili.

- E' stato fatto anche un tentativo di cercare un legame analitico piuttosto semplice tra $i$ valori di $\gamma$ trovati in questa fase preliminare e le corrispondenti profondità analitiche.

A tale scopo sono state ipotizzate due relazioni:

una lineare del tipo $m$

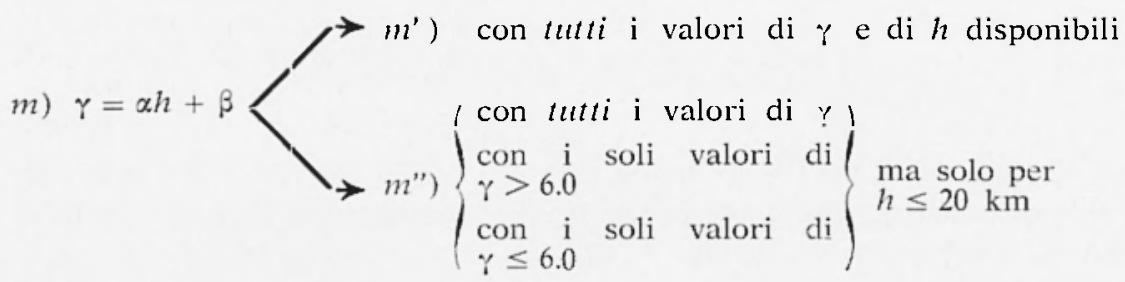


ed una del secondo ordine del tipo $n$

n) $\zeta=a+b h+c h^{2}\left\{\begin{array}{l}\text { con tutti } \text { i valori di } \% \\ \text { solo con } \gamma>6.0 \text { (ma escludendo } \gamma>>7.0) \\ \text { solo } \operatorname{con} \gamma<6.0\end{array}\right.$

La relazione che meglio raffigura il legame $\gamma=f(h)$ è ovviamente quella del tipo $n$, per quanto fino a circa $20 \mathrm{~km}$ di profondità anche l'andamento lineare è discretamente rappresentativo. Oltre i $20 \mathrm{~km}$ comunque sembrerebbe accettabile solo la $n$, che però, a sua volta, non è da ritenersi troppo indicativa per motivi che esporremo tra breve.

\subsection{Limiti di applicabilità del metodo}

L'insieme dei vari tentativi condotti per vie diverse, suggerisce l'opportunità di imporre dei limiti all'applicabilità del metodo. Questi limiti dovrebbero riguardare principalmente il numero delle isosiste da usare, la profondità dei terremoti e il valore di $\%$.

\section{1) Quanto alle isosiste}

Riteniamo il metodo applicabile solo entro una porzione ristretta dell'area epicentrale: suggeriamo di non dover oltrepassare l'uso della terza isosista. Si ricorda che le formule empiriche suggerite dai vari autori presuppongono una propagazione rettilinea dei raggi sismici, ed una distanza epicentrale confrontabile con un tratto rettilineo, onde poter supporre applicabile la geometria piana al triangolo Epicentro-Ipocentro-Osservatorio (rettangolo in Epic.).

Inoltre va tenuto presente che la formula di Blake, rispetto a quella di Kovesligethy presenta la diversità di trascurare il termine $\beta\left(D_{n}-h\right)$ il quale invece rappresenta un contributo notevole al valore della formula specie per $D_{n}$ abbastanza elevato. L'aver modificato nella [3"']

$$
\left(I_{v}-I_{n}\right)=3 \log \left(D_{n} / h\right)+\beta\left(D_{n}-h\right)
$$


il coefficiente fisso " 3 " in un coefficiente $\%$ variabile rende indubbiamente la formula applicabile ovunque con $\gamma$ appropriati, ma la rende inadeguata, a nostro avviso, per quanto riguarda valori di $D_{n}$ oltre un certo limite.

\section{2) Quanto alla profondità}

Riteniamo di dover limitare l'applicabilità del metodo solo ai terremoti con ipocentro entro la Crosta $(h \leq 33 \mathrm{~km})$. A profondità superiore cambiano le caratteristiche fisiche della Terra, per cui il valore di $\gamma$ (che nella formula proposta da Blake contiene in sé anche le attribuzioni fisiche spettanti più propriamente al coefficiente di assorbimento e di estinzione) dipenderebbe anche da tante altre variabili (densità, pressione, temperatura, stato chimico...) il cui legame è difficilmente rappresentabile in forma analitica. Entrare nel Mantello significa trovarsi di fronte ad incognite troppo importanti per azzardare un valore mediato di $\gamma$.

\section{3) Quanto al valore di $r$}

Riteniamo pertanto di dover imporre dei limiti anche ai valori di $\%$. I più immediati sono quelli che derivano dall'aver posto (nella [6] di Blake) $r$ variabile tra 0 e 1 , ossia $s$ ( $\%$ per noi) variabile fra 3 e 6 .

$\mathrm{Ne}$ risulta quindi che non è lecito servirsi della formula molto al di fuori del campo di variabilità suggerito dall'autore.

Poiché $Y$ risulta chiaramente variabile con $h$ riteniamo abbastanza lecito riferire ad esso alcune considerazioni riguardanti l'assorbimento.

Ricordiamo che l'assoríbimento dell'energia da parte del mezzo attraversato dalle onde sismiche può avvenire in due modi diversi (Caloi, 1978):

assorbimento proporzionale all'accelerazione della particella raggiunta dall'onda. Un tale assorbimento conduce ad una diminuzione della velocità delle onde longitudinali $\left(v_{1}\right)$ e trasversali $\left(v_{2}\right)$ relative ad un mezzo isotropo di densità $\rho$ secondo le seguenti relazioni:

$$
v_{1}=\left(\frac{\lambda+2 \mu}{\rho+k}\right)^{1 / 2} ; \quad v_{2}=\left(\frac{\mu}{\rho+k}\right)^{1 / 2}
$$


essendo $\mu$ e $\lambda$ le costanti di Lamé, e $k$ il coefficiente di proporzionalità che caratterizza la perdita di energia;

assorbimento proporzionale alla velocità della particella raggiunta dall'onda. Questa seconda ipotesi si verifica nel caso di un mezzo dispersivo definendo come tale un mezzo in cui la velocità dell'onda varia al variare del periodo.

Anclrebbe forse ritenuta più importante la prima ipotesi in quanto l'energia macrosismica in superficie, e gli effetti da essa prodotti, sono strettamente legati all'accelerazione. La velocità delle onde sismiche, e quindi l'accelerazione, dipende dunque anche dalla densità $\rho$ del mezzo $\mathrm{e}$, se il mezzo è assorbente, da $(\rho+k)$. Ma come si è detto $p$ varia al variare della profondita all'interno della Terra in quanto variano in maniera sconosciuta le condizioni fisiche di tensioni elastiche, di temperatura, di composizione chimica ecc....

Anche in un "modello" della Terra semplificato a strati concentrici omogenei e isotropi, separati da superfici di discontinuità, il gradiente di densità, supposto costante entro uno stesso strato, subisce variazioni nel passaggio da uno strato all'altro.

Il modello terrestre cosi semplificato consente agli studiosi una trattazione teorica del problema, però essi si rifiutano di estendere il modello alla Crosta, preferendo lavorare per semplicità sul modello della cosidetta "Terra sccrzata". Tra gli altri Bullen (1975) asserisce che "the structure of the Earth's crust is not only extremely complicated but is markedly different in different geographical areas".

Egli accetta (con riserva) di trattare anche la Crosta come uno strato omogeneo, ma solo in un panorama ampio di studio generale esteso a tutta la Terra, in modo che l'incidenza della Crosta possa ritenersi trascurabile. Quando però si restringe il campo della ricerca ad una porzione cosi piccola come l'Italia, non ì più lecito supporre lo strato crostale cmogeneo né la densità costante: e se poi ci si riferisce addirittura a stratificazioni sotiostanti la Moho, la cosa diventa ancora più complessa in quanto subentra, come detto, tutta una serie di parametri che incidono in maniera imprevedibile sulla legge di variazione di $p$.

A conclusioni analoghe si potrebbe giungere considerando il comportamento del "quality factor $Q$ ".

Ricordiamo che si chiama "fattore di qualità $Q$ " di un dato materiale attraversato da un'onda sismica, la costante

$$
Q=\frac{\pi}{\chi T \nu}
$$

essendo $\chi$ il coefficiente di assorbimento del materiale, $T$ e $v$ rispettivamente il periodo e la velocità di propagazione dell'onda sismica (Bath, 1973). 
Più basso è l'assorbimento, più alto è il fattore di qualità.

Le variazioni di $Q$ con la profondità all'interno della Terra sono state descritte da vari autori con risultati variabili dall'uno all'altro, peró le caratteristiche principali sono comuni.

All'interno della Crosta, fino a $30 \mathrm{~km}$, il valore di $Q$ si mantiene costante (circa 450 per le onde $P$ ): a $30 \mathrm{~km}$ subisce un brusco improv. viso calo verso un valore che si mantiene pressoché costante (circa 65) fin verso $60 \mathrm{~km}$. Oltre i $60 \mathrm{~km}$, nel Mantello, riprende ad aumentare.

Data la relazione $Q=\pi / \chi T v$ ne segue che, per lo stesso tipo di onda, l'assorbimento $\chi$ segue il processo inverso: pressoché costante fino a $30 \mathrm{~km}$, aumenta poi bruscamente e notevolmente per tornare a diminuire oltre i $60 \mathrm{~km}$.

Anche se un comportamento regolare del "fattore di qualità $Q$ " (e conseguentemente del coefficiente di assorbimento $\chi$ ) sia da ritenere solo ipotetico nella Crosta (giacché contrasta con tutte le evidenze sperimentali), resta tuttavia molto significativo il fatto del grandissimo aumento che subisce il coefficiente di assorbimento $\chi$ in corrispondenza del precipitare di $Q$ a circa $30 \mathrm{~km}$ di profondità.

Queste considerazioni tendono a giustificare la proposta da noi fatta di escludere dalla trattazione i terremoti con ipocentro sotto la Moho.

\subsection{Contenuto e analisi della Tabella 2}

Superata la fase preliminare e ritenendo motivati i limiti imposti siamo passati all'analisi sistematica i cui passi sono contenuti nella Tabella 2 . In essa i terremoti esaminati sono posti in ordine cronologico. Alcuni dei terremoti appartenenti alle categorie scartate (vedi il capitolo sulla scelta dei terremoti) sono stati riportati per rendere evidenti le anomalie cui conducono; però non sono stati considerati negli elaborati finali.

Le colonne da 1 a 6 riportano gli elementi base, secondo lo schema

data

(4) (5)

(6)

$(\phi, \lambda) \quad I 0 \quad h \pm \delta h$ 
I simboli che compaiono nella colonna delle intensità, sono quelli adottati per il Catalogo Sismico Nazionale dell'I.N.G. con il seguente significato:

$$
\begin{aligned}
& \mathrm{AK}=\text { l'intensità massima } \begin{array}{l}
\text { si è manifestata entro un'area molto } \\
\text { ristretta; di raggio } \leq 6 \mathrm{~km}
\end{array} \\
& \mathrm{AW}=\quad " \quad \begin{array}{l}
\text { potrebbe anche essere superiore a quel- } \\
\text { la indicata }
\end{array} \\
& \mathrm{BW}=\quad " \begin{array}{l}
\text { è quella individuata in località non epi- } \\
\text { centrale, per cui la } I_{\circ} \text { è certamente su- } \\
\text { periore a quella indicata. }
\end{array}
\end{aligned}
$$

A quest'ultima classificazione appartengono i terremoti del 29 agosto 1931 (con epicentro in mare, presso Istria); del 30 novem. 1934 (con epicentro in mare Adriatico)

che sono stati infatti scartati in un secondo tempo; e il terremoto del 13 novem. 1948 (con epicentro in mare, Nord Sardegna) sul quale però si è ritenuto lecito operare in quanto le isosiste, abbastanza valide, consentivano l'uso dei dati, ipotizzando ragionevolmente una $I_{0}=6.0+1.0(=7.0$ ? $)$. In questo caso i risultati dei calcoli sono stati posti tra parentesi.

Le colonne (6) e (7) riportano le profondità ipocentrali analitiche; nella colonna (6) come si è visto sono riportati anche i valori di $+\delta h$ quando lo studio originale li forniva; nella colonna (7) invece sono riportati solo i valori numerici delle profondità "scelte" per la ricerca in oggetto. Nella elaborazione dei dati infatti sono stati trascurati gli errori, che avrebbero complicato notevolmente i calcoli; ovviamente andrebbe tenuta presente l'incidenza che una tale omissione può comportare. In alcuni casi (Irpinia e Senigallia 1930; Veronese 1932...) sono stati considerati anche più valori di $h$, forniti da fonti informative diverse.

I limiti imposti alla profondità ipocentrale (da contenere entro la Crosta) hanno ridotto il numero di terremoti utilizzabili. Nella Tabella ne compaiono alcuni a conferma delle nostre argomentazioni. 
Le colonne da (8) a (13) contengono i successivi passi della elaborazione:

in particolare la (8) e la (9) riguardano le isosiste. [La colonna (8) contiene i gradi delle isosiste usate per ogni terremoto. Per i calcoli finali, come si è detto, sono stati assunti al massimo i primi tre. La colonna (9) riporta $\mathrm{i}$ valori in $\mathrm{km}$ dei raggi $\bar{r}_{c}$ dei cerchi equivalenti alle aree racchiuse dalle corrispondenti isosiste: nel caso di curve aperte i raggi sono stati ottenuti mediando su una serie di «misure », e i valori numerici sono posti tra parentesi].

Nella colonna (10) sono riportati i valori di $h$ in corrispondenza di $\gamma=3$ e $\gamma=6$ (usati da alcuni autori) da servire come termini di confronto. Nelle colonne (11) e (12) vengono riportati i risultati relativi alla ricerca del " $\because$ migliore" (vedi appresso).

Nella colonna (13) compaiono infine i $\gamma$ scelti per le operazioni finali. Sono indicati $\operatorname{con} C$ i terremoti appartenenti alla porzione di territorio indicata come Italia centrale (vedi figura 1).

2.5 La ricerca del $\gamma$ migliore. Analisi e discussione dei risultati

Partendo dall'assunto che la profondità più probabile di un terremoto sia quella ricavata analiticamente, chiameremo " $y$ migliore" il valore di " $\gamma$ " desunto in base alla conoscenza di essa.

Pertanto, per ogni terremoto della Tabella 2, utilizzando i valori di $I_{o}$, di $h$ analitica, e di $\vec{r}_{c}$, sono stati calcolati i valori dei $\because$, relativamente a ciascuna delle isosiste disponibili, facendo uso della formula [8]. I risultati sono posti nella colonna (11).

La colonna (12) riporta i valori mediati

$$
\bar{\gamma}=\frac{\Sigma \gamma_{i}}{i} \pm \delta \gamma
$$

L'esame dei risultati offre lo spunto per alcune interessanti considerazioni:

- va notato come i valori di $\bar{\gamma}$ non siano troppo dissimili da quelli calcolati con le sole seconde isosiste $\left(I_{o}-I_{1}\right)=2$; per 


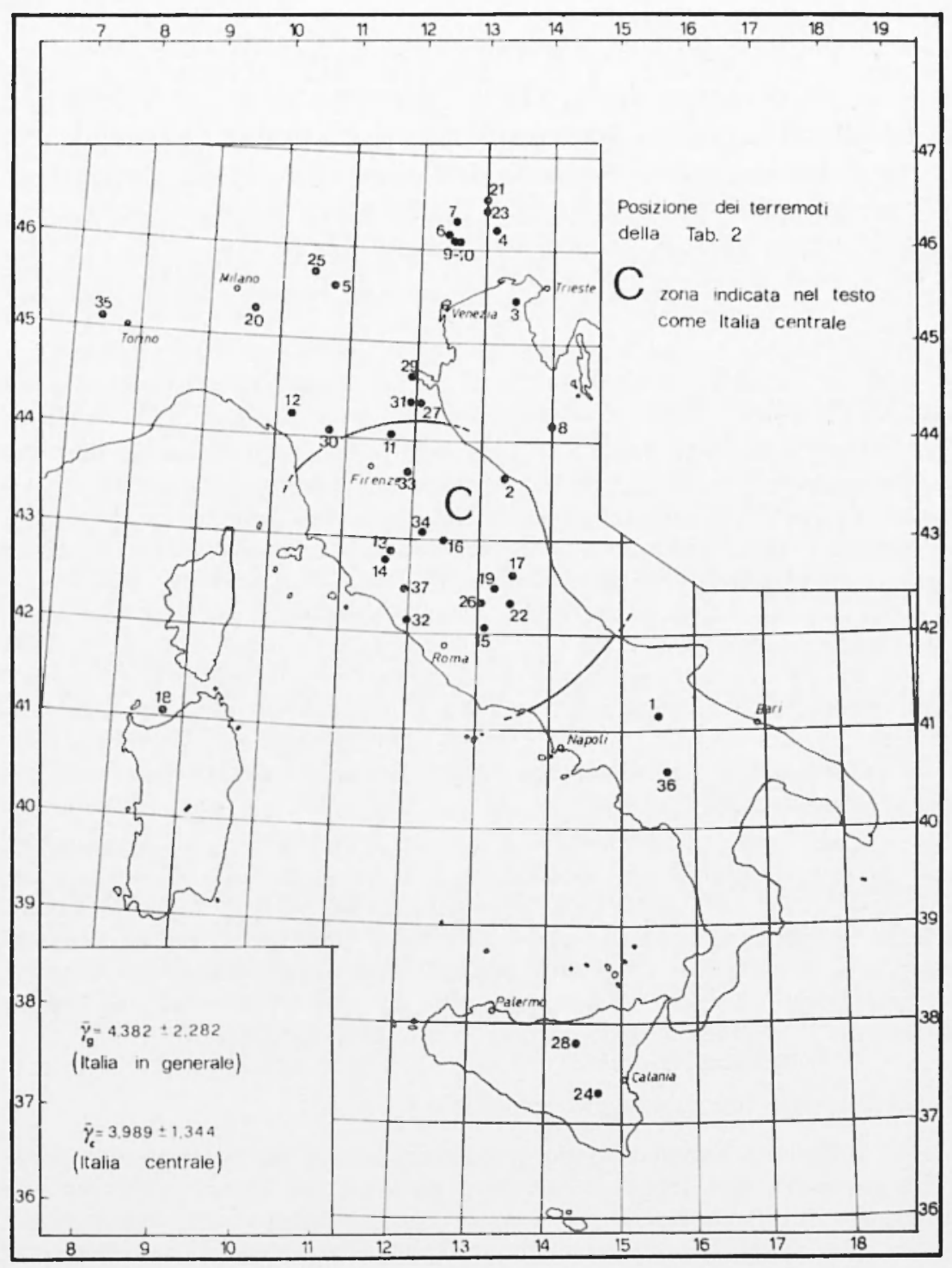

Fig. 1 - Posizione dei terremoti dalla Tabella 2. 
cui, per calcoli rapidi, potrebbe ritenersi abbastanza lecito servirsi solo di tali isosiste;

- si noti ancora come il modo di variare del $\gamma$, pur contenuto entro il campo di variabilità piuttosto ristretto impostogli, si manifesti alquanto imprevedibile: è indubbia la sua dipendenza dalla struttura del mezzo attraversato dalle onde sismiche;

- alcune forti deviazioni standard di esso si accoppiano spesso a forti deviazioni $+\delta h$ (anche se per la valutazione del " $\gamma$ migliore" è stato preso in considerazione solo il valore "pulito" di $h$ ).

\section{Discussione su alcuni $\gamma$}

.. Il Y della Val Padana risulta molto basso, ma questo non dovrebbe stupire: la Val Padana presenta un comportamento indubbiamente anomalo nel quadro generale del resto dell'Italia.

Stupisce invece alquanto il basso valore che si è trovato nel Gran Sasso (5 settembre 1950) ed a Tolmezzo (Arta) (5 novembre 1956).

Per il Gran Sasso si potrebbe discutere sulla probabilità che la $I_{0}$ sia maggiore dell'VIII grado riscontrato dalle notizie: l'area di VIII infatti è molto estesa e si può presumere che all'epicentro l'intensità massima possa aver raggiunto, in zona disabitata, un grado più elevato (VIII 1/2 - IX). In tale ipotesi i raggi delle isosiste sarebbero spostati e il valore del $Y$ aumenterebbe, pur mantenendosi su valori bassi: passerebbe da 1.962 a 2.367.

- Invece per il terremoto di Arta non si può dire nulla. Si potrebbe solo mettere in dubbio la profondità di $2 \mathrm{~km}$, ma nulla di particolare autorizza a ciò; tuttavia sarebbe bene tener presente che quando i calcoli analitici conducono a valori molto piccoli di profondità, questi sono da considerarsi indicativi di una $h$ generalmente contenuta entro $\mathrm{i}$ primi $10 \mathrm{~km}$. Questo è anche quanto ribadito da vari ricercatori che hanno affrontato il problema delle piccole profondità ipocentrali.

Y nei distretti (un sondaggio orientativo)

E' sufficiente l'analisi condotta sulla Tabella 2 per desumere la difficoltà di trarre una legge di carattere generale sul legame effettivo che intercorre tra il coefficiente $\%$ e la profondita ipocentrale. Forse, come si era già accennato, si potrebbe giungere a risultati più soddisfacenti

1) qualora si disponesse di un gran numero di dati di terremoti distribuiti in maniera uniforme su tutto il territorio italiano,

2) qualora i dati di profondità fossero forniti con un alto grado di attendibilità, 

sola $(*)$.

3) qualora fosse ben definita la carta sismo-tettonica della peni-

Il comportamento del $\%$ potrebbe allora rappresentare una caratteristica degli eventuali distretti sismici.

Abbiamo rinunciato a malincuore al progetto di associare i $\gamma$ ai vari distretti. Un rapido sondaggio orientativo condotto servendoci delle 19 unità tettonico-stratigrafiche del Modello Strutturale dell'Italia pubblicato nel 1975 (CNR) fa intravedere la possibilità di migliorare i risultati ottenuti nella presente ricerca associando al valore medio generale di $\bar{i}$ un termine correttivo $+\delta \bar{\gamma}$ specifico per ogni distretto.

Non riportiamo i valori trovati poiché scarsamente affidabili, ma il metodo generale, applicato su basi statisticamente informative, dovrebbe condurre a risultati interessanti quanto al comportamento strutturale delle singole zone.

\subsection{Il valore di $\bar{\gamma}$ proposto per l'Italia in generale $\left(\bar{\gamma}_{g}\right)$}

Pertanto, nella impossibilità materiale di operare al momento in maniera diversa, ci siamo serviti dei risultati ottenuti per proporre un valore medio di $\bar{\gamma}$ ricercato su tutto il territorio italiano: il "range" di variabilità generale comprenderà ovviamente gli eventuali "range" minori e diversi, dovuti ad eventuali frazionamenti.

L'operazione, condotta su tutti i terremoti che compaiono nella colonna (13) della Tabella 2, ha portato a stabilire il seguente valore da applicare, in generale, a tutta l'Italia:

$$
\bar{\gamma}_{g}=4.382 \pm 2.282 \text { (per l'Italia in generale) }
$$

il che comporta un $\vec{\gamma}_{g}$ variabile tra

$$
\left(\bar{\gamma}_{g}\right) \min =2.100 \quad \text { e }\left(\overline{\gamma_{g}}\right) \max =6.664
$$

(*) Questo programma è in studio da parte del Progetto Finalizzato Geodinamica del C.N.R. 
2.7 Il valore di $\bar{\gamma}$ proposto per l'Italia centrale $\left(\bar{\gamma}_{1}\right)$

Una seconda media è stata calcolata considerando solo i terremoti dell'Italia centrale (indicati con $C$ nella Tabella 2 e nell'acclusa carta d'Italia): l'Italia centrale ha fornito materiale più numeroso perché è stata oggetto di vari studi particolareggiati.

Il $\bar{\gamma}$ proposto per questa zona risulta essere:

$$
\bar{\gamma}_{c}=3.989+1.344 \quad \text { (per l'Italia centrale) }
$$

il che comporta un $\bar{\gamma}_{c}$ variabile tra

$$
\left(\vec{\gamma}_{c}\right) \min =2.645 \text { e }\left(\bar{\gamma}_{c}\right) \max =5.333
$$

\section{CRITICHE}

A questo punto si può ragionevolmente obiettare che non è lecito mediare aritmeticamente su valori legati tra loro in maniera tanto complessa e per di più legati ad altri parametri di cui è ignoto il vincolo analitico; ma visto quanto siano ancora perfettibili anche i risultati analitici, in particolare la profondità ipocentrale, si possono accettare i risultati ottenuti tenendo presente, ripetiamo, che dalla trattazione macrosismica non si potrà mai pretendere la perfezione. In mancanza di dati analitici però, anche un parametro macrosismico calcolato con una approssimazione ragionevole, può fornire un'utile informazione.

4. TABElla 3 Per L'APPlicazione immediata del METOdo

Accettati per validi i risultati ottenuti, è stata calcolata la Tabella 3 numerica per l'uso pratico e immediato del metodo. Conoscendo i $r_{c}$ della prima, o della seconda, o della terza isosista, si ha immediatamente il valore di $h+\delta h$. 
L'intervallo di variabilità \pm oh è stato calcolato tenendo conto del campo di variabilità del $\bar{\gamma}$

$$
(\bar{\gamma} \pm \delta \bar{\gamma})
$$

sia per l'Italia in generale, sia per l'Italia centrale.

Ovviamente è consigliabile mediare sui valori ottenuti da tutte e tre le isosiste: per una valutazione grossolana ancor più rapida si può tener presente quanto già osservato, che il valore singolo che più si avvicina alla media è quello relativo alla seconda isosista.

5. LE PROFONDITÀ MACROSISMICHE POSTE A CONFRONTO CON QUELLE ANALITICHE

Ricerca di $h$ in funzione dei $\bar{Y}$ trovati; $h=f(\bar{\gamma})$ e scarti relativi

A questo punto della ricerca abbiamo voluto operare un confronto tra le profondità analitiche e le stesse profondità dedotte invece macrosismicamente tenendo conto dei $\gamma$ trovati.

E' ovvio che si opera entro un circuito chiuso, però questa verifica può servire a valutare l'entità degli scarti relativi all'uso del " $r$ medio" anziché all'uso del " $r$ migliore".

A tale scopo abbiamo ricalcolato le profondità dei terremoti della Tabella 2 (escludendo da questa verifica $h>33 \mathrm{~km}$ ) servendoci dei due valori di $r$ trovati

$$
\begin{array}{ll}
\gamma_{g}=4.382 & \text { (Italia in generale) } \\
\gamma_{c}=3.989 & \text { (Italia centrale) }
\end{array}
$$

e degli $\vec{r}_{c}$ delle prime, seconde e terze isosiste dei terremoti della Tabella 2.

Per ciascun terremoto sono stati trovati tre valori di $h$ : $h_{1} \rightarrow$ usando solo la prima isosista $\left(I_{0}-I_{n}\right)=1 ;($ oppure $=1.5)$ $h_{2} \rightarrow$ usando solo la seconda isosista $\left(I_{o}-I_{n}\right)=2$; $($ oppure $=2.5)$ $h_{3} \rightarrow$ usando solo la terza isosista $\left(I_{o}-I_{n}\right)=3 ;($ oppure $=3.5)$ 
La media aritmetica dei tre valori di $h$ di ciascun terremoto è poi stata posta a confronto con la profondità analitica.

La Tabella 4 compendia questi calcoli.

Anche qui si ritrova come, in generale, il valore della profondità macrosismica calcolata con la sola seconda isosista sia molto prossimo a quello calcolato come media fra le tre isosiste.

Nelle ultime due colonne della Tabella 4 sono riportate le differenze

$$
\varepsilon=\left(h_{\text {anal }}-h_{\text {macr }}\right)
$$

calcolate con il $\bar{\gamma}_{g}$ per tutti i terremoti esaminati, e con il $\bar{\gamma}_{c}$ per i terremoti ubicati nella zona cosi indicata (zona C).

Gli errori più grandi si ritrovano, naturalmente, per i terremoti 20 e 21 di cui si è già discusso: per tutti gli altri il divario è inferiore a $|10| \mathrm{km}(*)$.

Nel grafico di figura 2 è resa visibile la posizione delle $h$ macrosismiche (in ordinate) rispetto a quelle analitiche (in ascisse).

La situazione ottimale è rappresentata ovviamente dall'equazione della bisettrice

retta (a) $\quad h_{\text {anal. }}=h_{\text {macr. }}$

Le rette (b) e (c) del grafico rappresentano i limiti di variabilità delle profondità ipocentrali in corrispondenza del campo di variabilità $(\gamma \mp \delta \gamma)(* *)$.

In questa panoramica solo i terremoti di Deruta $(h=3 \mathrm{~km}$; n. 16) e del Gran Sasso ( $h=3.2 \mathrm{~km}$; n. 19) resterebbero al di fuori, ma ricordiamo quanto si è detto sui valori molto piccoli di $h$.

(*) Escludiamo da questa analisi il terremoto n. 13. La sua profondità, calcolata analiticamente, comportava già in partenza un errore molto elevato: $h=(27 \pm 16.7) \mathrm{km}$.

$\left({ }^{* *}\right)$ Per semplicità le rette $(\mathrm{b})$ e $(\mathrm{c}),\left(\dot{r}_{\iota_{\text {anal }}}=h_{\text {macr. }}+\delta h\right)$ sono state tracciate riferendosi solo ai valori di $(h \mp \delta h)$ relativi alle seconde isosiste, calcolati con il $\bar{\gamma}_{g}$. 


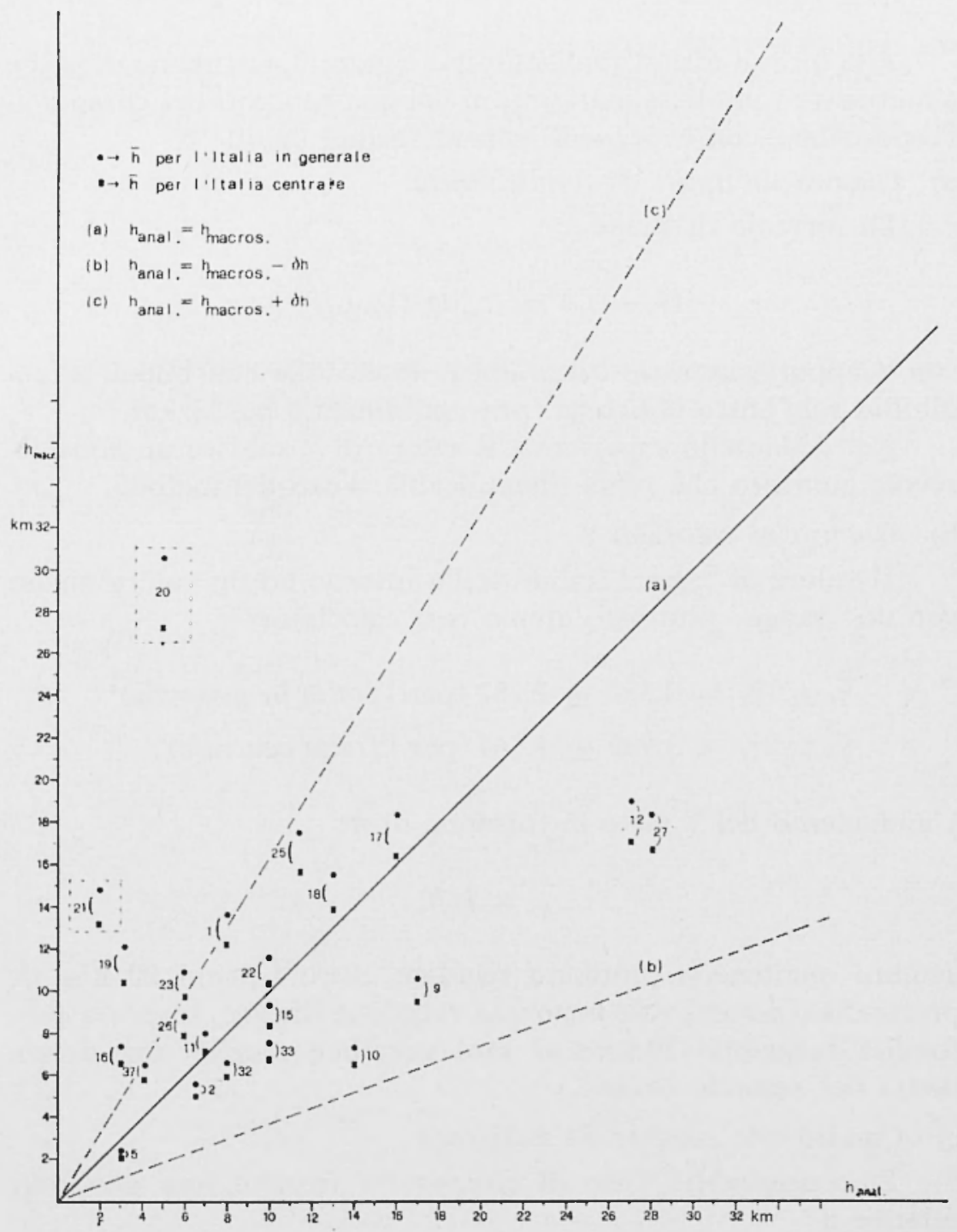

Fig. 2 - Confronto tra gli $h_{\text {anal }}$ e gli $h_{\text {macr }}$ effettuato con i valori di $h+o h$ relativi alla seconda isosista (con il $\bar{Y}_{g}$ ). 


\section{CONSIDERAZIONI FINALI}

Alla luce di tutta l'analisi fin qui condotta, riteniamo di poter sintetizzare i punti salienti, alcuni dei quali toccati nel corso dell'esposizione, con le seguenti considerazioni finali:

a) Quanto ai limiti di applicabilità

La formula di Blake

$$
\left(I_{0}-I_{n}\right)=\gamma \log \left(D_{n} / h\right)
$$

con $\gamma$ opportunamente calcolato, è applicabile con buona attendibilità solo entro la Crosta (presumibilmente $h \leq 33 \mathrm{~km}$ ).

Nel "Mantello superiore " il valore di $\gamma$ subisce un considerevole aumento che rende inapplicabile l'uso del metodo.

b) Quanto ai valori $d i \gamma$

Il valore di $\bar{\gamma}$ per l'Italia oscilla intorno ad un valore medio con un "range" piuttosto ampio così calcolato:

$$
\begin{aligned}
& \bar{\gamma}_{B} \pm \delta \bar{\gamma}_{g}=4.382 \pm 2.282 \text { (per l'Italia in generale) } \\
& \bar{\gamma}_{c} \pm \delta \bar{\gamma}_{c}=3.989 \pm 1.344 \text { (per l'Italia centrale) }
\end{aligned}
$$

L'andamento del $\bar{\gamma}$ visto in funzione di $h$

$$
\bar{\gamma}=f(h)
$$

sembra mantenersi piuttosto regolare entro i primi $20 \mathrm{~km}$. di profondità, per cui vale bene una relazione lineare. Invece a profondità maggiori $(20 \leq h \leq 30 \mathrm{~km})$ varrebbe meglio una dipendenza del secondo ordine.

c) Quanto alle isosiste da utilizzare

E' consigliabile l'uso di almeno tre isosiste (ma non più) relative a

$$
\left(I_{\mathrm{o}}-I_{n}\right)=1 ; \quad\left(I_{\mathrm{o}}-I_{n}\right)=2 ; \quad\left(I_{\mathrm{o}}-I_{n}\right)=3
$$

e dei corrispondenti $\overline{r_{c}}$. 
Volendo utilizzarne una sola è da preferire la seconda.

La Tabella 3 è stata compilata con questo intendimento.

L'uso di isosiste di grado molto basso andrebbe evitato, potendo, per difficoltà di demarcarne ragionevolmente i confini.

Si ponga attenzione che qualora si disponga di isosiste del tipo

$$
\left(I_{\circ}-I_{n}\right)=\quad \begin{aligned}
& 1.5 \\
& 2.5 \\
& 3.5
\end{aligned}
$$

occorre procedere ai calcoli corrispondenti (non si può far uso immediato della Tabella 3 ). 


\section{BIBLIOGRAFIA}

B̈̈rı M., 1973 - Introduction to Seismology. Birkhäuser Verlag, Basel and Stutigart.

B.AкI: ARchis, 1941 - On the estimation of focal Depth from macroseismic data. "Bull. Seism. Soc. Amer."

But.l.:N K.E., 1975 - The Earth's Density. Chapman and Hall, London.

Calor P., 1978 - La terra e i terremoti. I vol. "Introduzione alla Sismologia ". Teorie, melodi, strumenti. "Accademia Nazionale dei Lincei", Roma.

Cancanı A., 1904 . Sur l'emploi d'une double èchelle sismique des intensilés, empirique et absclute. Relazione presentata alla II Conferenza Internazionale di Sismologia, Lipsia.

Gnssmann F., 1925 - Die makroseismischen Intensitäten der schweizerischen Nahebeben... "Jahresber. d. Schweiz. Erdbebendienstes », Zürich.

Grablivitz G., 1910 - Sullaccelerazione sismica. "Boll. Soc. Sism. Ital.", XIV.

Kövest.ıgithy (de) F., 1906 - Seismonomia. "Boll. Soc. Sism. Ital. ", XI.

Jinosy Eм., 1906 - Bestimmung der Herdtiefe und des seismichen Absorptions-Koefficienten des Charlestoner Erabebens. "Boll. Soc. Sism. Ital. ", XI.

Marcillt. L., Peronaci M., 1979 - Analisi critica su le profondilà ipocentraii. "Annali di Geofisica", XXXII (volume unico).

Structural Modil. cF Italy, 1975 - Quaderni de "La Ricerca Scientifica " del Consiglio Nazionale delle Ricerche, Roma. 


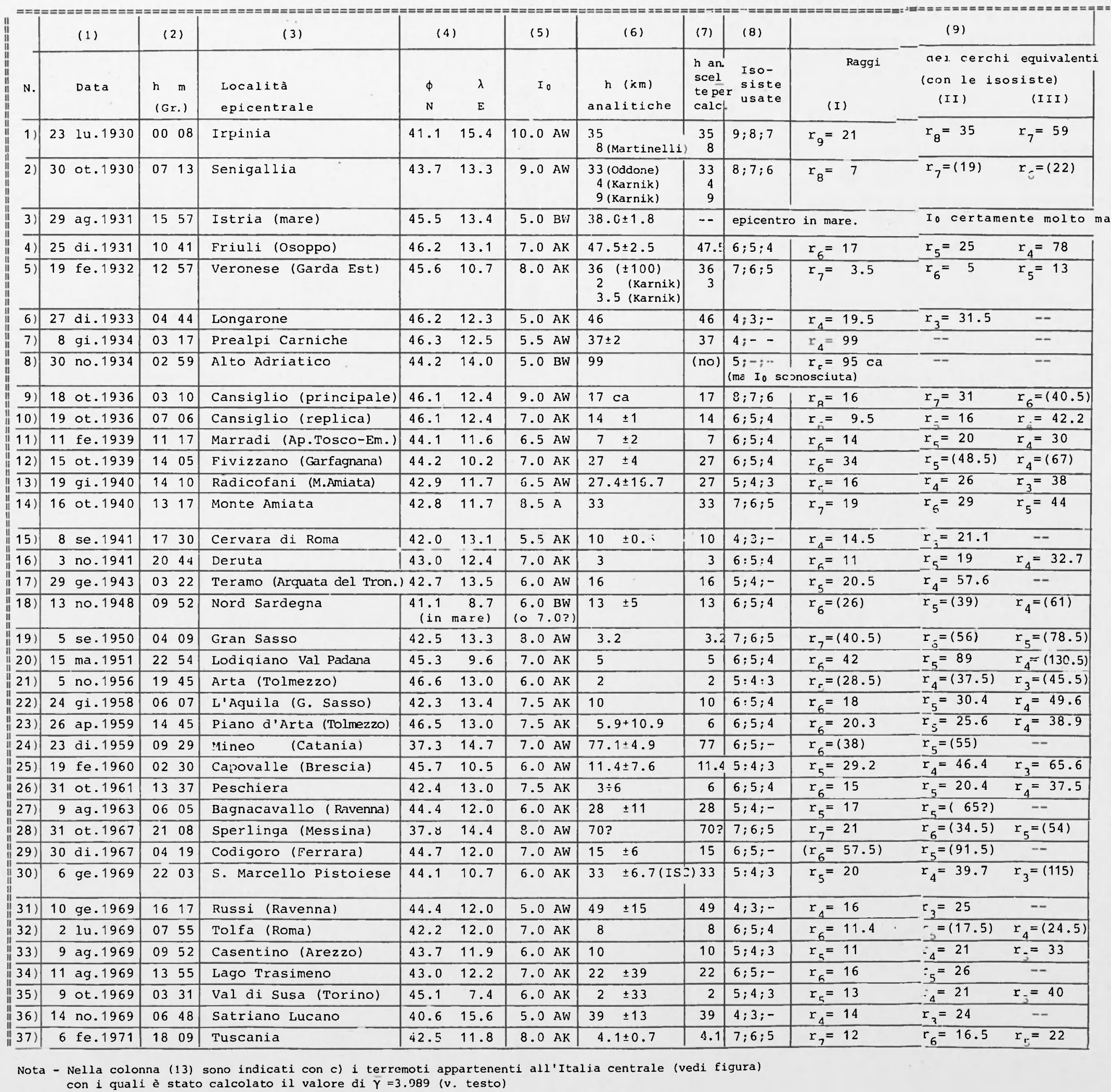

Nota - Nella colonna (13) sono indicati con c) i terremoti appartenent
con $\mathbf{i}$ quali è stato calcolato il valore di $Y=3.989$
(v. testo)

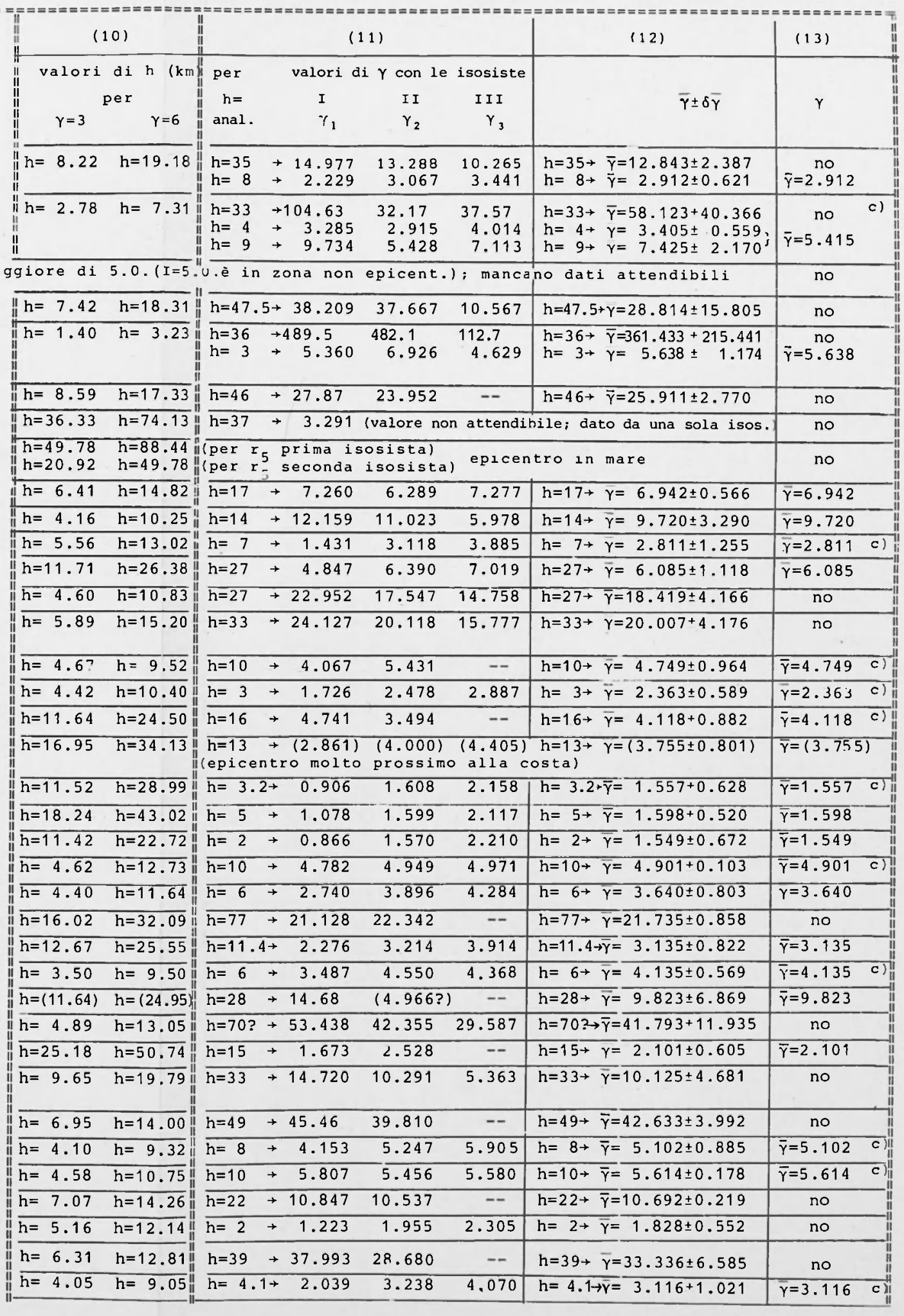


T A B E L L A 3

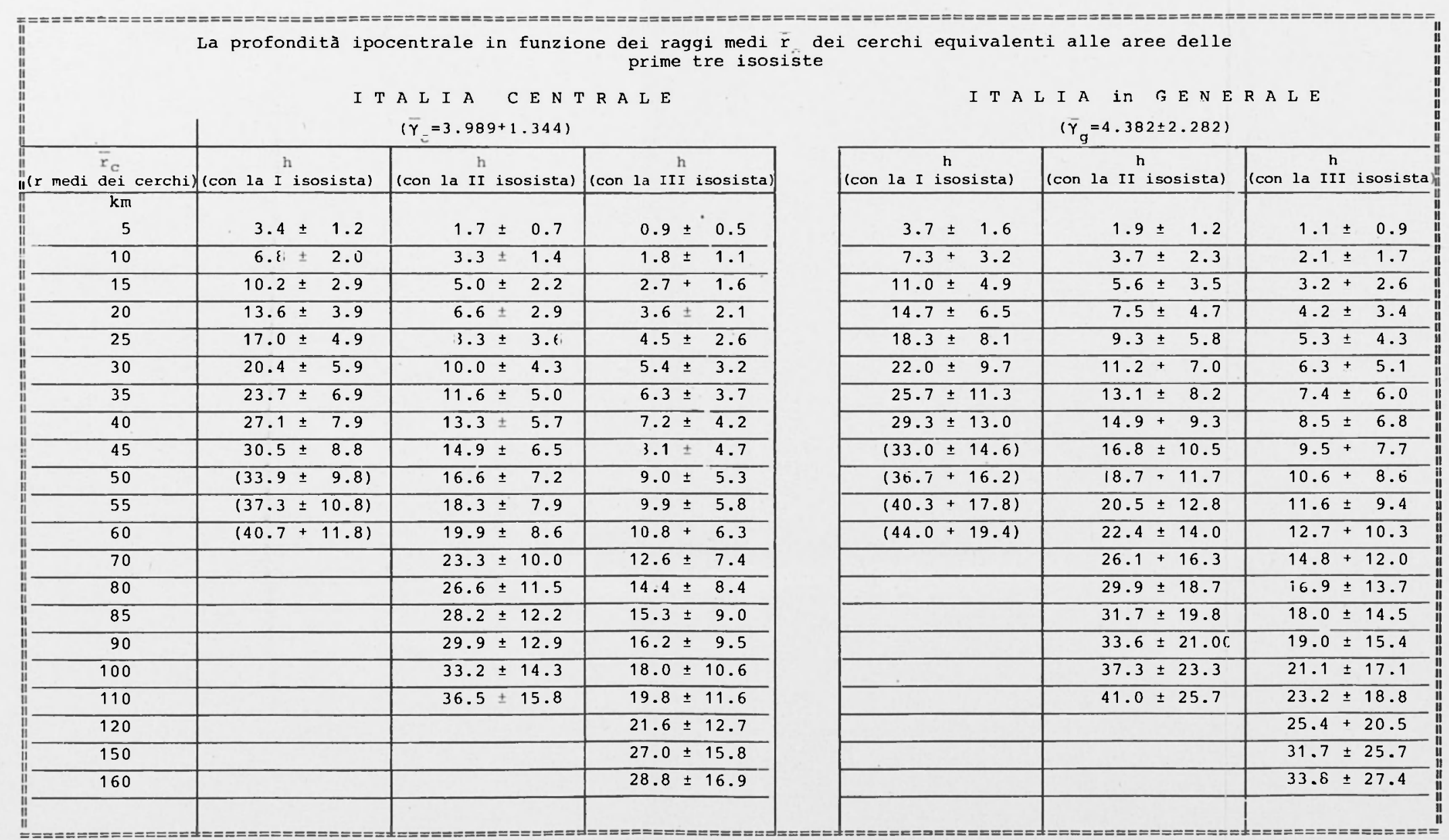


TABELLA 4

Italia in generale $(\bar{\gamma}=4.382)$

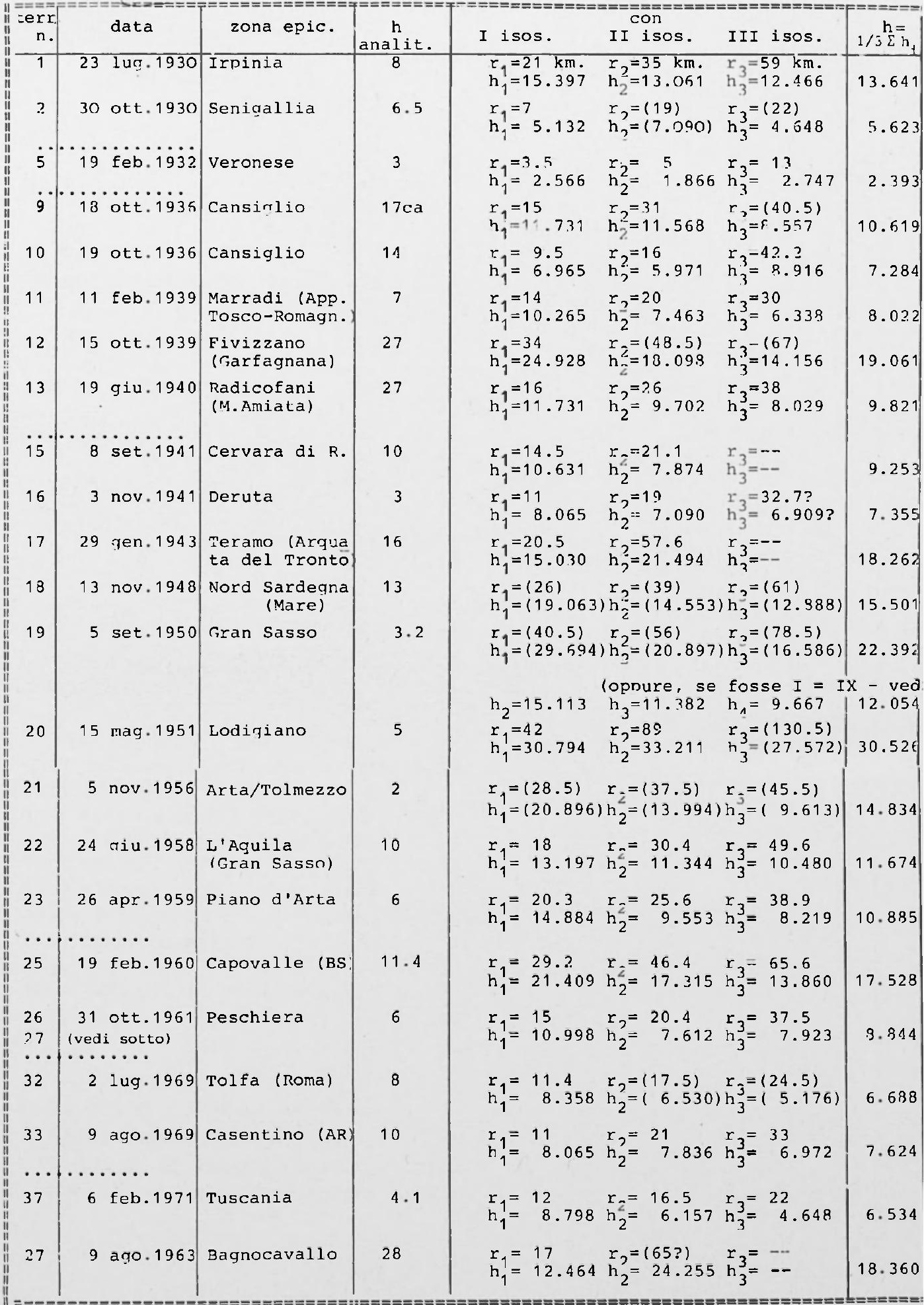


Italia centrale $(\bar{y}=3.989)$

\begin{tabular}{|c|c|c|c|}
\hline I isos. & II & III is. & $1 / 3 \sum h_{i}$ \\
\hline 14.248 & 11.626 & 10.609 & 12.161 \\
\hline $4.74^{\circ}$ & 5.311 & 3.956 & 5.005 \\
\hline 2.375 & 1.661 & 2.338 & 2.125 \\
\hline 10.856 & 10.297 & 7.283 & 9.479 \\
\hline 6.446 & 5.315 & 7.588 & 6.450 \\
\hline 9.499 & 6.643 & 5.395 & 7.179 \\
\hline 23.058 & 16.110 & 12.048 & 17.075 \\
\hline 10.856 & 8.636 & 6.833 & 8.775 \\
\hline 9.838 & 7.009 & -- & 8.424 \\
\hline 7.463 & 6.311 & $5.880 ?$ & 6.551 \\
\hline 13.909 & 19.132 & $\cdots$ & 16.521 \\
\hline 17.640 & 12.954 & 10.469 & 13.854 \\
\hline 27.478 & 18.601 & 14.116 & 20.065 \\
\hline $\begin{array}{l}\text { zes }=0) \\
13.452\end{array}$ & 10.070 & 10.807 & 10.443 \\
\hline 28.496 & 29.562 & 23.467 & 27.175 \\
\hline 19.337 & 12.456 & 8.182 & 13.325 \\
\hline 12.213 & 10.098 & 8.919 & 10.410 \\
\hline 13.773 & 8.503 & 6.995 & 9.757 \\
\hline 19.816 & 15.412 & 11.796 & 15.675 \\
\hline 10.171 & 6.776 & 6.743 & 7.897 \\
\hline 7.735 & 5.813 & 4.406 & 5.985 \\
\hline 7.463 & 6.975 & 5.934 & 6.791 \\
\hline 8.142 & 5.481 & 3.956 & 5.860 \\
\hline 11.534 & 21.590 & -- & 16.562 \\
\hline
\end{tabular}

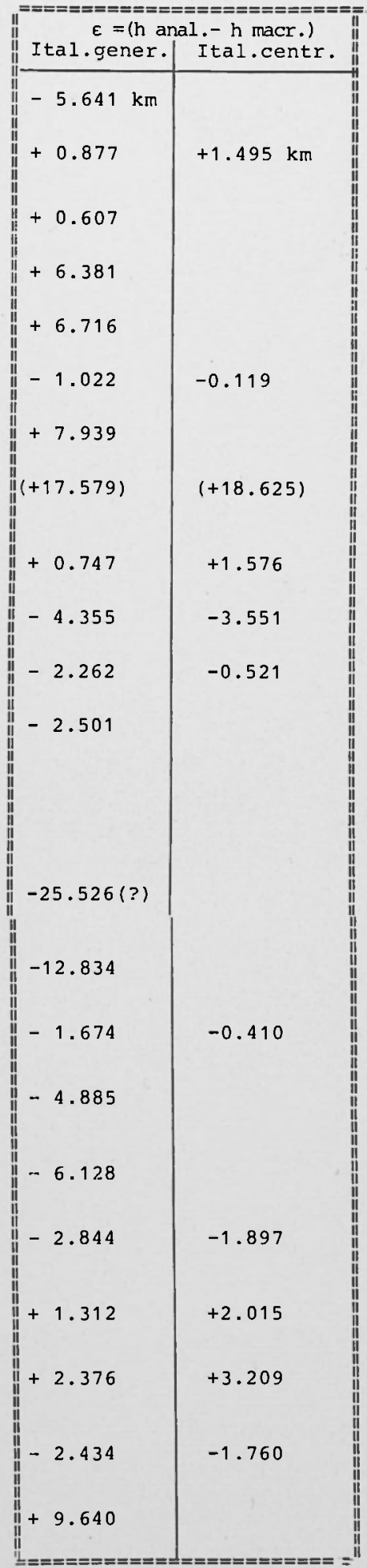

